\title{
The Effect of Remote Training during Covid-19 Quarantine on Body Composition Changes in Collegiate Football Players
}

\author{
Lee Stowers* and José R Fernández \\ Department of Nutrition Sciences, University of Alabama at Birmingham, USA \\ *Corresponding author: Lee Stowers, Department of Nutrition Sciences, University of Alabama at \\ Birmingham, USA
}

\begin{abstract}
Background: Previous research has shown that following a structured training plan can result in significant changes in body composition in elite football players. However, the effect on body composition changes after athletes complete the same training plan remotely with limited resources needs further investigation.
\end{abstract}

Aim: The purpose of this study is to determine if body composition changes in collegiate football players differ after training remotely during COVID-19 quarantine compared to training on campus during a routine off-season training period.

Methods: This study was conducted as a secondary analysis of routine data collection in a southeastern collegiate athletic department. Body composition data was collected during off-season training in two separate groups of collegiate football players. Group 1 data was collected before and after the COVID-19 quarantine forced athletes to replicate their training remotely. Group 2 data was collected before and after a typical off-season training program on campus. Changes in body composition over the training period were calculated for each group and analyzed for statistical differences.

Results: Anova was used to analyze the changes in weight, body fat, muscle mass, and BMl changes between groups. Results showed a significant change in muscle mass between groups 1 and $2(F=2.53, p=0.0012)$.

Conclusion: These results suggest that training remotely may not be as effective in building muscle mass when compared to traditional on campus training. Additionally, on campus resources such as coaches, support staff, nutrition, and community training may influence training results.

\section{Keywords}

Body Composition, Remote training, COVID-19, Football

\begin{abstract}
Abbreviations
BIA: Bioelectrical Impedance Analysis; BMI: Body Mass Index; IRB: Institutional Review Board; NCAA: National Collegiate Athletic Association; U.S: United States
\end{abstract}

\section{Introduction}

Collegiate football players in the National Collegiate Athletic Association (NCAA) division 1 participate in a maximum of 8 or 20 hours of weekly in-person sports specific activities [1], depending on off- or in-season, respectively. On campus during the off-season (JanuaryJuly), football athletes typically spend 6-8 weeks training prior to the start of spring practice. The off-season is mainly focused on training to prepare for the physical demands of in-season [2]. Many universities have larger football training facilities and coaching staffs working specifically with football teams [3] that include athletic trainers, dietitians, and mental health professionals.

On March 12, 2020, the NCAA began cancelling sporting events in response to the COVID-19 pandemic spreading in the United States (U.S.) resulting in a nationwide quarantine [4]. Collegiate football players retained remote access to training programs and support staff during this time. The shutdown of training facilities increased the difficulty of recreating a typical preseason training program resulting in a reduction in training volume and intensity [5]. Additionally, quarantine was associated with an increase in daily sitting time of three hours and deteriorating eating habits with significant increase in carbohydrate intake 
[6]. The reduction in physical activity and diet quality resulting from the COVID-19 quarantine could be detrimental for body composition by increasing body fat and decreasing muscle mass [7].

Previous research has shown significant changes in body composition can occur in elite football players following structured nutrition and training plans for a variety of time periods $[2,8]$. Increases in lean mass and decreases in total body fat in collegiate football players have been shown to increase power, strength, and speed across all position groups [2]. Prior research has shown that a halt in training of as little as 2-4 weeks can result in a loss of strength and performance [7]. These losses occur regardless of intensity of previous exercise regimen and increase as absence of training continues [7]. It is not known if significant body composition changes can be replicated in football players training remotely during quarantine.

The experience of a pandemic presented the opportunity to evaluate the impact of remote training in collegiate athletes. Athletes were expected to follow training plans to the best of their abilities despite losing the physical presence of training partners, coaches, and support staff, along with supplemental food regularly provided by the nutrition team. The purpose of this study is to determine if body composition changes in collegiate football players differ after training remotely during COVID-19 quarantine compared to training on campus during a routine off-season training period. Due to changes in training and loss of resources, we hypothesize that there will be a significant difference in changes in body fat and muscle mass between groups.

\section{Methods}

\section{Study design and participants}

This study was secondary analysis of data previously collected in a southeastern collegiate athletic department as routine care. This study was approved by the Institutional Review Board (IRB) and utilized data from one hundred and thirty-one collegiate football players. Body composition was measured pre and post training cycle for 2 separate groups. Group 1 consisted of 36 football players who trained remotely over quarantine. Group 2 consisted of 95 football players who completed a typical 8-week spring training program on the university campus. Those eligible for inclusion were medically cleared for athletic participation from the Athletics Sports Medicine Department. Those who had not completed initial body composition testing prior to COVID-19 or were not cleared for active sport participation were ineligible. All athletes were National Collegiate Athletic Association (NCAA) Division I football players between the ages 18-23. Mean age, race, and height of each group, along with other characteristics, are described in Table 1.

\section{Study protocol}

Body composition measurements were conducted as a part of standard of care protocol. Athletes in group 1 reported to the athletic training facility for an initial body composition assessment (Visit 1 ) at the conclusion of offseason football training in February 2020. After this assessment, the COVID-19 pandemic resulted in a 3-month shut-down of all athletic facilities and activities. During this shutdown, athletes were asked to complete prescribed strength and conditioning plans remotely to their best ability. Strength and conditioning plans were individually prescribed by the director of football athletic performance and based on athletes' access to gym equipment. When athletics activities resumed in June 2020, athletes completed a follow-up body composition assessment (Visit 2) within two weeks of their return. Body composition and weight were measured using a multi-current, segmental BIA device.

Athletes in group 2 reported to the athletic training facility for an initial body composition assessment (Visit 1) at the beginning of the spring 2021 semester prior to beginning preseason training. All athletes completed a standard 8-week offseason strength and conditioning program on campus at the athletic facility. Athletes had full access to the nutrition station during this time where they could obtain food pre and post workout. The second body composition measurement (Visit 2)

Table 1: Group participant characteristics $(\mathrm{N}=131)$.

\begin{tabular}{|l|l|l|}
\hline & $\begin{array}{l}\text { Group 1 } \\
\text { (N=36) }\end{array}$ & $\begin{array}{l}\text { Group 2 } \\
\text { (N = 95) }\end{array}$ \\
\hline Characteristic & Mean & Mean \\
\hline Age (yr) & $20.50 \pm 1.34$ & $20.58 \pm 1.41$ \\
\hline Race & & \\
\hline White & $44.44 \%$ & $29.47 \%$ \\
\hline Black & $55.56 \%$ & $70.53 \%$ \\
\hline Height (m) & $1.88 \pm 0.07$ & $1.86 \pm 0.04$ \\
\hline Weight (kg) & $107.44 \pm 24.45$ & $103.87 \pm 22.49$ \\
\hline Baseline BMI & 29.79 & 29.78 \\
\hline Baseline Fat Mass (kg) & $21.55 \pm 15.41$ & $21.9 \pm 14.13$ \\
\hline Baseline Muscle Mass & $81.36 \pm 10.02$ & $77.95 \pm 9.07$ \\
(kg) & & \\
\hline Position group (\%) & & $17.89 \%$ \\
\hline Offensive line & $33.33 \%$ & $10.53 \%$ \\
\hline Wide Receivers & $16.67 \%$ & $5.26 \%$ \\
\hline Tight Ends & $16.67 \%$ & $7.37 \%$ \\
\hline Running Backs & $11.11 \%$ & $5.26 \%$ \\
\hline Quarterbacks & $11.11 \%$ & $8.42 \%$ \\
\hline Linebackers & $5.56 \%$ & $21.05 \%$ \\
\hline Defensive Backs & $2.78 \%$ & $5.26 \%$ \\
\hline Special Teams & $2.78 \%$ & $11.58 \%$ \\
\hline Defensive Line & $0 \%$ & $7.37 \%$ \\
\hline Outside Linebackers & $0 \%$ & \\
\hline & & \\
\hline
\end{tabular}


was conducted with all athletes at the conclusion of the training program. Body composition and weight were measured using a multi-current, segmental BIA device.

\section{Body composition and weight analysis}

All weight and body composition measurements were taken using a Tanita MC-780U (Tanita Corp of America, Inc. Arlington Heights, Illinois, USA), an 8-mode segmental BIA system that has 3 assessment frequencies $(5 \mathrm{kHz} / 50 \mathrm{kHz} / 250 \mathrm{kHz})$ and 1 measurement current (up to $90 \mu \mathrm{A}$ ) [9]. All athletes were reminded to follow standard of care daily hydration protocol prior to measurement. All assessments were taken in the morning prior to training. Athletes were required to remove shoes and socks and wear light clothing for each measurement. Each participant stepped on to the scale with toes and heels placed on the electrodes of the weighing platform. Whole body fat mass, fat-free mass (i.e. lean mass), percent body fat, and total body water were recorded.

\section{Statistical analysis}

Four continuous variables were analyzed including changes in total body fat mass, body fat percent, muscle mass, and Body Mass Index (BMI). Change was calculated for all variables by obtaining the difference of visit 1 from visit 2 . All data was normally distributed. Data was determined to have equal variances by results of the Levene's test. A variable (repeat) was created to represent athletes who were in both groups to determine if this caused an effect on results. A one-way ANOVA was used to compared mean differences for each dependent variable between groups. Repeat athletes, age, race, position, and height were all assessed as covariates in all models. Additional adjustments were made to body fat (MM change), muscle mass (BF Percent), and BMI (MM change, BF Percent). Analyses were conducted using SAS (Version 9.4) and an alpha level of $p<0.05$ for statistical significance.

\section{Results}

The results of our four statistical models, after adjusting for covariates are shown in Table 2. Model 1 $(F=6.11, p<0.0001)$ evaluated body weight changes between groups. Although there were no significant differences in weight change between groups, significant contributions were observed in change in muscle mass between groups and position. Our second statistical model $(F=1.71, p=0.0424)$ evaluated change in body fat between groups, results showed no

Table 2: Analysis of variance for body composition changes between groups.

\begin{tabular}{|c|c|c|c|c|c|}
\hline Model & $\begin{array}{c}\text { Dependent } \\
\text { Variable }\end{array}$ & $\begin{array}{l}\text { Independent } \\
\text { Variable }\end{array}$ & Covariate & F-Value & P-Value \\
\hline \multirow[t]{6}{*}{1} & Body weight & Group & MM Change & 61.17 & $<0.0001$ \\
\hline & & & Age & 1.29 & 0.2660 \\
\hline & & & Race & 0.59 & 0.4459 \\
\hline & & & Position & 2.15 & 0.0310 \\
\hline & & & Height & 0.28 & 0.5968 \\
\hline & & & Group & 2.00 & 0.1604 \\
\hline \multirow[t]{6}{*}{2} & Body Fat & Group & MM Change & 8.32 & 0.0047 \\
\hline & & & Age & 1.19 & 0.3166 \\
\hline & & & Race & 1.43 & 0.2349 \\
\hline & & & Position & 2.65 & 0.0080 \\
\hline & & & Height & 0.48 & 0.4912 \\
\hline & & & Group & 3.02 & 0.0850 \\
\hline \multirow[t]{6}{*}{3} & Muscle Mass & Group & BF Percent & 12.69 & 0.0005 \\
\hline & & & Age & 0.33 & 0.9188 \\
\hline & & & Race & 0.38 & 0.5380 \\
\hline & & & Position & 2.99 & 0.0032 \\
\hline & & & Height & 1.28 & 0.2610 \\
\hline & & & Group & 4.25 & 0.0416 \\
\hline \multirow[t]{7}{*}{4} & BMI & Group & BF Percent & 95.51 & $<0.0001$ \\
\hline & & & MM Change & 83.56 & $<0.0001$ \\
\hline & & & Age & 0.64 & 0.7018 \\
\hline & & & Race & 0.23 & 0.6342 \\
\hline & & & Position & 1.42 & 0.1901 \\
\hline & & & Height & 0.40 & 0.5267 \\
\hline & & & Group & 0.88 & 0.3508 \\
\hline
\end{tabular}


significant difference. However, change in muscle mass and position group were shown to contribute to change in body fat. The results of the statistical model 3 ( $F=$ 2.53, $p=0.0012$ ), which evaluated muscle mass changes between groups, after adjusting for covariates showed there was a significant difference in muscle mass gains during training periods between groups. Body fat percent and position group were shown to contribute to this significant change. The results of the final statistical model ( $F=10.26, p=<0.0001$ ) evaluating $\mathrm{BMI}$, resulted in no significant differences between groups. Although we did not see a significant difference, body fat percent and muscle mass change were shown to make significant contributions to change in BMI. Age, race, and height were not shown to make significant contributions to changes in body weight, body fat, muscle mass, or BMI between groups.

\section{Discussion}

While there were no significant differences observed in body weight, body fat, or BMI our results partially support our hypothesis showing a significant difference in muscle mass between training styles. Group 2 who trained on campus experienced greater changes in muscle mass that the group who trained remotely. This agrees with previous longitudinal studies on body composition in collegiate football players which have shown significant increases in muscle mass during the offseason [2]. These results suggest that changes in workout environment influence on training outcomes. To our knowledge, this is the first study to evaluate body composition changes in collegiate football players upon completion of 3 months of remote off-season training while quarantined.

The reasons underlying this change in muscle mass are not clear, but research in multisport athletes during quarantine showed that multiple factors during this natural event decreased exercise intensity and increasing sedentary behaviors. Since exercise training has been shown to promote positive body composition changes, reduced exercise intensity and increased sedentary behavior may explain our findings [10]. Previous longitudinal studies in collegiate football players have shown an increase in exercise intensity resulted in gains in muscle mass and reduction of body fat [2]. Mental health during this unprecedented time may have also contributed to this intensity shift. Athletes surveyed during this time reported training alone for the majority of quarantine instead of training with others in person or virtually [11]. This decrease in teammate interaction may have had negative effect on accountability and explain why more than half reported training alone at lower intensity $[5,11,12]$. Multiple surveys have shown that student athletes who remained connected to their teammates during quarantine, reported lower levels of depression than those who did not $[12,13]$. One in two athletes that experienced depression during this time expressed that this resulted in a lack of motivation to train and increased sedentary behavior. Athletes also experienced a decrease in human interaction outside of athletics with classes also being forced to be online. This would likely increase sedentary behavior even further because daily walking around campus to classes, lunch, work, etc, were halted. Since, sedentary behavior can be detrimental to physical and mental health [12] all these factors mentioned may have contributed to our results.

While sedentary behavior increase was a major factor of this natural event, another resource that athletes lost access too was the football nutrition station. During normal circumstances, players have daily access to protein shakes, sandwiches, fruit, and snacks before and after workouts and meet with their dietitian daily. Athletes are on a limited scholarship income, so they may not have been able to afford to financially compensate for this reduction of food access which could have resulted in major daily dietary changes during quarantine. Restricted access to food has been shown to decrease diet quality with substitution of unhealthy foods and increased fast food intake under normal circumstances [14]. Since body fat was not increased as expected, nutrition knowledge and Registered Dietitian (RD) support may have had an influence on results. While this study did not assess nutrition knowledge directly, none of the players in the quarantine group were new to the team and had been previously educated on the importance of a balanced diet and their specific nutrition requirements for optimal health and training recovery by their team RD. All players retained remote access to their RD during this time who was able to answer any nutrition questions and help athletes with food access resources if necessary. Previous research has shown that athletes with greater nutrition knowledge were more likely to be near their protein and carbohydrate goals in their daily intake [15]. Nutrition knowledge has also been shown to have a positive correlation with fatfree mass in athletes [15] Nutrition attitudes also have an influence in dietary intake and may have been altered during this unprecedented time [1]. Overall, loss of nutrition access may have influenced body composition, but because of previous nutrition knowledge and RD access during quarantine, changes to body fat and BMI may not have been significantly different during this time as previously expected.

\section{Limitations}

This study is limited by sample size and specificity of the sample (male collegiate football players). Due to the unpredictable nature of the COVID-19 pandemic, we have limited information on athletes' workout conditions, nutrition knowledge, dietary intake, finances, or mental health during this time. Athletes were brought back in phases because of return to campus protocol after quarantine causing group 1 to have fewer athletes than group 2. As a result, only 7 position groups were 
represented in group 1 compared to all 9 in group 2 . Considering that position group played a significant contribution to our results in muscle mass and body fat changes, inability to represent all position groups may have affected baseline numbers and outcomes. While both training periods were considered pre-season training. Covid caused the two training cycles that were compared in this study to be at two slightly different times of year. During a normal year, athletes would have advanced to the next phase of preseason training before quarantine ended. This extension of this training cycle may have affected outcomes. Additionally, body composition was measured using BIA which is highly sensitive to hydration and electrolyte changes [16-18]. Results could have been affected by athlete's hydration status.

\section{Conclusion}

In conclusion, athletes who trained on campus gained more muscle mass than athletes who trained remotely. These findings suggest that training on campus with a team and access to equipment, facilities, and support staff may increase efficacy of exercise. This could be attributed to the accountability, intensity, and community training with a team and coach brings. Further research studying the effects remote training and limited food access may have on athletes are needed, particularly with how virtual training affects physical and mental health.

\section{Acknowledgements}

The study was designed by L. Stowers and J.F. Fernandez and data were collected by L. Stowers; the data were analyzed L. Stowers and J.R. Fernandez; and data interpretation and manuscript preparation were undertaken by L. Stowers and J.R. Fernandez. All authors approved the final version of the paper. The authors have no conflicts of interest in this study. Additional contributions in manuscript development were made by Paula C. Chandler-Laney. The authors would also like to thank the Nutrition and Obesity Research Center at the University of Alabama at Birmingham and Bill Clark for support during development of this publication.

\section{References}

1. Current student-Athletes (2020).

2. Trexler ET, Smith-Ryan AE, Mann JB, Ivey PA, Hirsch KR, et al. (2017) Longitudinal Body Composition Changes in NCAA Division I College Football Players. J Strength Cond Res 31: 1-8.

3. Judge LW, Petersen JC, Bellar DM, Craig BW, Cottingham MP, et al. (2014) The current state of NCAA Division I collegiate strength facilities: Size, equipment, budget, staffing, and football status. J Strength Cond Res 28: 22532261.

4. Smeyers (2020) NCAA cancels remaining winter and spring championships.
5. Mon-López D, de la Rubia Riaza A, Hontoria Galán M, Refoyo Roman I (2020) The Impact of Covid-19 and the Effect of Psychological Factors on Training Conditions of Handball Players. Int J Environ Res Public Health 17: 6471.

6. Ammar A, Brach M, Trabelsi K, Chtourou H, Boukhris O, et al. (2020) Effects of COVID-19 Home Confinement on Eating Behaviour and Physical Activity: Results of the ECLB-COVID19 International Online Survey. Nutrients 12: 1583.

7. Jukic I, Calleja-González J, Cos F, Cuzzolin F, Olmo J, et al. (2020) Strategies and Solutions for Team Sports Athletes in Isolation due to COVID-19. Sports (Basel, Switzerland) 8: 56.

8. Roberta A, Johnathan OM (2015) Football Player Body Composition: Importance of Monitoring for Performance and Health.

9. Delk-Licata A, Behrens CE, Benardot D, Bertrand BM, Chandler-Laney PC, et al. (2019) The Association between Dietary Protein Intake Frequency, Amount, and State of Energy Balance on Body Composition in a Women's Collegiate Soccer Team. Int J Sports Exerc Med 5: 123.

10. Ormsbee MJ, Arciero PJ (2012) Detraining Increases Body Fat and Weight and Decreases VO2 peak and Metabolic Rate. J Strength Cond Res 26: 2087-2095.

11. Pillay $L$, Janse van Rensburg DCC, Jansen van Rensburg A, Ramagole DA, Holtzhausen L, et al. (2020) Nowhere to hide: The significant impact of coronavirus disease 2019 (COVID-19) measures on elite and semi-elite South African athletes. J Sci Med Sport 23: 670-679.

12. Graupensperger S, Benson AJ, Kilmer JR, Evans MB (2020) Social (Un) distancing: Teammate Interactions, Athletic Identity, and Mental Health of Student-Athletes During the COVID-19 Pandemic. J Adolesc Health 67: 662-670.

13. Praharso NF, Tear MJ, Cruwys T (2017) Stressful life transitions and wellbeing: A comparison of the stress buffering hypothesis and the social identity model of identity change. Psychiatry Res 247: 265-275.

14. Morales ME, Berkowitz SA (2016) The Relationship between Food Insecurity, Dietary Patterns, and Obesity. Curr Nutr Rep 5: 54-60.

15. Devlin BL, Leveritt MD, Kingsley M, Belski R (2017) Dietary Intake, Body Composition, and Nutrition Knowledge of Australian Football and Soccer Players: Implications for Sports Nutrition Professionals in Practice. Int J Sport Nutr Exerc Metab 27: 130-138.

16. Castizo-Olier J, Irurtia A, Jemni M, Carrasco-Marginet $M$, Fernández-García R, et al. (2018) Bioelectrical impedance vector analysis (BIVA) in sport and exercise: Systematic review and future perspectives. PLoS One 13: e0197957.

17. Chen $P$, Mao L, Nassis GP, Harmer P, Ainsworth BE, Li $F$ (2020) Coronavirus disease (COVID-19): The need to maintain regular physical activity while taking precautions. $J$ Sport Health Sci 9: 103-104.

18. Moonen HPFX, van Zanten FJL, Driessen L, de Smet V, Slingerland-Boot R, et al. (2020) Association of bioelectric impedance analysis body composition and disease severity in COVID-19 hospital ward and ICU patients: The BIAC-19 study, Clinical Nutrition. 